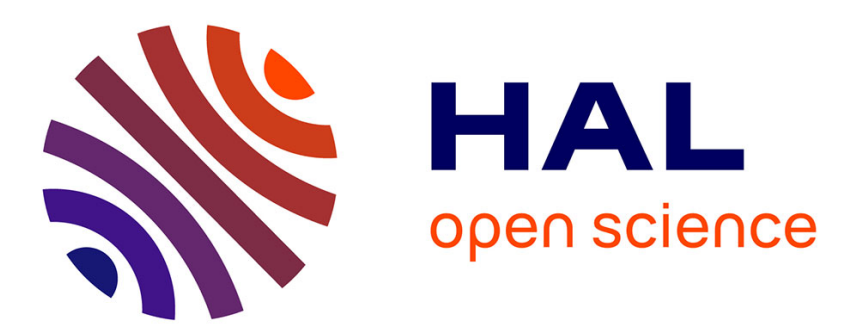

\title{
On the Hybrid Cramér-Rao Bound and Its Application to Dynamical Phase Estimation
}

Stéphanie Bay, Benoit Geller, Alexandre Renaux, Jean-Pierre Barbot, Jean-Marc Brossier

\section{- To cite this version:}

Stéphanie Bay, Benoit Geller, Alexandre Renaux, Jean-Pierre Barbot, Jean-Marc Brossier. On the Hybrid Cramér-Rao Bound and Its Application to Dynamical Phase Estimation. IEEE Signal Processing Letters, 2008, 15, pp.453-456. 10.1109/LSP.2008.921461 . hal-01239443

HAL Id: hal-01239443

https://hal-ensta-paris.archives-ouvertes.fr/hal-01239443

Submitted on 7 Dec 2015

HAL is a multi-disciplinary open access archive for the deposit and dissemination of scientific research documents, whether they are published or not. The documents may come from teaching and research institutions in France or abroad, or from public or private research centers.
L'archive ouverte pluridisciplinaire HAL, est destinée au dépôt et à la diffusion de documents scientifiques de niveau recherche, publiés ou non, émanant des établissements d'enseignement et de recherche français ou étrangers, des laboratoires publics ou privés. 


\title{
On the Hybrid Cramér-Rao bound and its application to dynamical phase estimation
}

\author{
Stéphanie Bay, Benoit Geller, Alexandre Renaux, Member, IEEE, Jean-Pierre Barbot and Jean-Marc
}

Brossier

\begin{abstract}
This letter deals with the Cramér-Rao bound for the estimation of a hybrid vector with both random and deterministic parameters. We point out the specificity of the case when the deterministic and the random vectors of parameters are statistically dependent. The relevance of this expression is illustrated by studying a practical phase estimation problem in a non data-aided communication context.
\end{abstract}

\section{INTRODUCTION}

A natural problematic when designing an estimator is the evaluation of its performance. Lower bounds on the Mean Square Error (MSE) mainly answer this question and the well known Cramér-Rao Bound (CRB) is widely used by the signal processing community. Depending on assumptions on the parameters, the CRB has different expressions. When the vector of parameters is assumed to be deterministic, we obtain the standard CRB [1] and when the vector of parameters is assumed to be random with an a priori probability density function (pdf), we obtain the so-called Bayesian CRB [2].

At the end of the eighties, an extension combining both the standard and the Bayesian CRBs has been proposed [3]. Indeed, in some practical scenarios, it is natural to represent the parameter vector by a deterministic part and by a random part. This bound has thus been called the Hybrid CRB (HCRB) and a nice tutorial can be found in [4]. Until now, results available in the literature essentially focussed on the case where the deterministic part and the random part of the parameter vector are assumed to be statistically independent (see, e.g., Eqn. (5) in [3], Eqn. (13)

Stéphanie Bay and Jean-Pierre Barbot are with the Ecole Normale Supérieure de Cachan, SATIE Laboratory, 61, avenue du President Wilson 94235 Cachan, France. (e-mail: \{bay,barbot\}@ satie.ens-cachan.fr)

Benoit Geller is with Ecole Nationale Supérieure des Techniques Avancées, Laboratory of Electronics and Computer Engineering (LEI), 32, Boulevard Victor 75015 Paris, France. (e-mail: geller@ensta.fr)

Alexandre Renaux is with University Paris-Sud 11, Laboratory of Signals and Systems, Supélec, 3 rue Joliot-Curie, 91192 Gif-sur-Yvette cedex, France. (e-mail: renaux@1ss.supelec.fr)

Jean-Marc Brossier is with Grenoble Institute of Technology, GIPSA Laboratory, 961 rue de la Houille Blanche Domaine universitaire - BP 4638402 Saint Martin d'Hères cedex, France. (e-mail: jean-marc.brossier@gipsa-lab.inpg.fr)

This work was supported in part by the French ANR (Agence Nationale de la Recherche), LURGA project and by the European network of excellence NEWCOM++. 
in [4] and Eqn. (13) in [5]). To the best of our knowledge, a closed-form expression of the HCRB with a statistical dependence between the deterministic and the random parameters has never been reported in the literature. The goal of this paper is then twofold. First, in Section II, we remind the structure of the HCRB and we point out the specificity of the case when the deterministic part and the random part of the parameter vector are statistically dependent. Second, in Section III, motivated by this analysis we give a closed-form expression of the proposed bound in the practical case of a dynamical phase subject to a linear drift in a non data-aided communication context.

\section{THE HYBRID CRAMÉR-RAO BOUND}

\section{A. Background}

Let $\boldsymbol{\mu}=\left(\boldsymbol{\mu}_{r}^{T} \boldsymbol{\mu}_{d}^{T}\right)^{T} \in \mathbb{R}^{n}$ be the parameter vector that we have to estimate. This vector is split into two subvectors $\boldsymbol{\mu}_{d}$ and $\boldsymbol{\mu}_{r}$ where $\boldsymbol{\mu}_{d}$ is assumed to be a $(n-m) \times 1$ deterministic vector and $\boldsymbol{\mu}_{r}$ is assumed to be a $m \times 1$ random vector with an a priori pdf $p\left(\boldsymbol{\mu}_{r}\right)$. The true value of $\boldsymbol{\mu}_{d}$ will be denoted $\boldsymbol{\mu}_{d}^{\star}$. We consider $\hat{\boldsymbol{\mu}}(\mathbf{y})$ as an estimator of $\boldsymbol{\mu}$ where $\mathbf{y}$ is the observation vector. The HCRB satisfies the following inequality on the MSE

$$
\mathbb{E}_{\mathbf{y}, \boldsymbol{\mu}_{r} \mid \boldsymbol{\mu}_{d}^{\star}}\left[\left.(\hat{\boldsymbol{\mu}}(\mathbf{y})-\boldsymbol{\mu})(\hat{\boldsymbol{\mu}}(\mathbf{y})-\boldsymbol{\mu})^{T}\right|_{\boldsymbol{\mu}_{d}^{\star}}\right] \geq \mathbf{H}^{-1}\left(\boldsymbol{\mu}_{d}^{\star}\right),
$$

where $\mathbf{H}\left(\boldsymbol{\mu}_{d}^{\star}\right) \in \mathbb{R}^{n \times n}$ is the so-called Hybrid Information Matrix (HIM) defined as [3]

$$
\mathbf{H}\left(\boldsymbol{\mu}_{d}^{\star}\right)=\mathbb{E}_{\mathbf{y}, \boldsymbol{\mu}_{r} \mid \boldsymbol{\mu}_{d}^{\star}}\left[-\left.\Delta_{\boldsymbol{\mu}}^{\boldsymbol{\mu}} \log p\left(\mathbf{y}, \boldsymbol{\mu}_{r} \mid \boldsymbol{\mu}_{d}\right)\right|_{\boldsymbol{\mu}_{d}^{\star}}\right]
$$

where $\left[\Delta_{\boldsymbol{\eta}}^{\nu}\right]_{k, l}=\frac{\partial^{2}}{\partial[\boldsymbol{\eta}]_{k} \partial[\boldsymbol{\nu}]_{l}}$.

When the deterministic and the random parts of the parameter vector are assumed to be independent, and after some algebraic manipulations, the HIM can be rewritten as (see [4], Eqn. (18))

$$
\mathbf{H}\left(\boldsymbol{\mu}_{d}^{\star}\right)=\mathbb{E}_{\boldsymbol{\mu}_{\boldsymbol{r}}}\left[\mathbf{F}\left(\boldsymbol{\mu}_{d}^{\star}, \boldsymbol{\mu}_{r}\right)\right]+\left(\begin{array}{cc}
\mathbb{E}_{\boldsymbol{\mu}_{\boldsymbol{r}}}\left[-\Delta_{\boldsymbol{\mu}_{r}}^{\boldsymbol{\mu}_{r}} \log p\left(\boldsymbol{\mu}_{r}\right)\right] & \mathbf{0}_{m \times(n-m)} \\
\mathbf{0}_{(n-m) \times m} & \mathbf{0}_{(n-m) \times(n-m)}
\end{array}\right),
$$

where

$$
\mathbf{F}\left(\boldsymbol{\mu}_{d}^{\star}, \boldsymbol{\mu}_{r}\right)=\mathbb{E}_{\mathbf{y} \mid \boldsymbol{\mu}_{d}^{\star}, \boldsymbol{\mu}_{r}}\left[-\left.\Delta_{\boldsymbol{\mu}}^{\boldsymbol{\mu}} \log p\left(\mathbf{y} \mid \boldsymbol{\mu}_{d}, \boldsymbol{\mu}_{r}\right)\right|_{\boldsymbol{\mu}_{d}^{\star}}\right]
$$

With this aforementioned structure, it is straightforward to reobtain the standard and the Bayesian CRBs. Indeed, if $\boldsymbol{\mu}=\boldsymbol{\mu}_{d}$, we have

$$
\mathbf{H}^{-1}\left(\boldsymbol{\mu}_{d}^{\star}\right)=\left(\mathbb{E}_{\mathbf{y} \mid \boldsymbol{\mu}_{d}^{\star}}\left[-\left.\Delta_{\boldsymbol{\mu}_{d}}^{\boldsymbol{\mu}_{d}} \log p\left(\mathbf{y} \mid \boldsymbol{\mu}_{d}\right)\right|_{\boldsymbol{\mu}_{d}^{\star}}\right]\right)^{-1}
$$

which is the standard CRB, and, if $\boldsymbol{\mu}=\boldsymbol{\mu}_{r}$, we have

$$
\mathbf{H}^{-1}=\left(\mathbb{E}_{\mathbf{y}, \boldsymbol{\mu}_{r}}\left[-\Delta_{\boldsymbol{\mu}_{r}}^{\boldsymbol{\mu}_{r}} \log p\left(\mathbf{y} \mid \boldsymbol{\mu}_{r}\right)\right]+\mathbb{E}_{\boldsymbol{\mu}_{r}}\left[-\Delta_{\boldsymbol{\mu}_{r}}^{\boldsymbol{\mu}_{r}} \log p\left(\boldsymbol{\mu}_{r}\right)\right]\right)^{-1},
$$

which is the Bayesian CRB. 


\section{B. Extension when $\boldsymbol{\mu}_{r}$ and $\boldsymbol{\mu}_{d}$ are statistically dependent}

We now assume a possible statistical dependence between $\boldsymbol{\mu}_{r}$ and $\boldsymbol{\mu}_{d}$. In other words, $\boldsymbol{\mu}_{r}$ is now assumed to be a $m \times 1$ random vector with an a priori pdf $p\left(\boldsymbol{\mu}_{r} \mid \boldsymbol{\mu}_{d}^{\star}\right) \neq p\left(\boldsymbol{\mu}_{r}\right)$.

Based on the HIM definition given by Eqn. (2) and expending the log-likelihood as $\log p\left(\mathbf{y}, \boldsymbol{\mu}_{r} \mid \boldsymbol{\mu}_{d}^{\star}\right)=\log p\left(\mathbf{y} \mid \boldsymbol{\mu}_{d}^{\star}, \boldsymbol{\mu}_{\boldsymbol{r}}\right)+$ $\log p\left(\boldsymbol{\mu}_{r} \mid \boldsymbol{\mu}_{d}^{\star}\right)$, we obtain the following HIM

$$
\mathbf{H}\left(\boldsymbol{\mu}_{d}^{\star}\right)=\mathbb{E}_{\boldsymbol{\mu}_{r} \mid \boldsymbol{\mu}_{d}^{\star}}\left[\mathbf{F}\left(\boldsymbol{\mu}_{d}^{\star}, \boldsymbol{\mu}_{r}\right)\right]+\mathbb{E}_{\boldsymbol{\mu}_{r} \mid \boldsymbol{\mu}_{d^{\star}}}\left[-\left.\Delta_{\boldsymbol{\mu}}^{\boldsymbol{\mu}} \log p\left(\boldsymbol{\mu}_{r} \mid \boldsymbol{\mu}_{d}\right)\right|_{\boldsymbol{\mu}_{d}^{\star}}\right],
$$

where $\mathbf{F}\left(\boldsymbol{\mu}_{d}^{\star}, \boldsymbol{\mu}_{r}\right)$ is given by Eqn. (4).

In order to explicitly show the modification in comparison with the HIM given by Eqn. (3), $\mathbf{H}\left(\boldsymbol{\mu}_{d}^{\star}\right)$ can be rewritten as follows

$$
\mathbf{H}\left(\boldsymbol{\mu}_{d}^{\star}\right)=\mathbb{E}_{\boldsymbol{\mu}_{r} \mid \boldsymbol{\mu}_{d}^{\star}}\left[\mathbf{F}\left(\boldsymbol{\mu}_{d}^{\star}, \boldsymbol{\mu}_{r}\right)\right]+\mathbb{E}_{\boldsymbol{\mu}_{r} \mid \boldsymbol{\mu}_{d}^{\star}}\left[\left(\begin{array}{cc}
-\Delta_{\boldsymbol{\mu}_{r}}^{\boldsymbol{\mu}_{r}} \log p\left(\boldsymbol{\mu}_{r} \mid \boldsymbol{\mu}_{d}^{\star}\right) & -\left.\Delta_{\boldsymbol{\mu}_{d}}^{\boldsymbol{\mu}_{r}} \log p\left(\boldsymbol{\mu}_{r} \mid \boldsymbol{\mu}_{d}\right)\right|_{\boldsymbol{\mu}_{d}^{\star}} \\
\left(-\left.\Delta_{\boldsymbol{\mu}_{d}}^{\boldsymbol{\mu}_{r}} \log p\left(\boldsymbol{\mu}_{r} \mid \boldsymbol{\mu}_{d}\right)\right|_{\boldsymbol{\mu}_{d}^{\star}}\right)^{T} & -\left.\Delta_{\boldsymbol{\mu}_{d}}^{\boldsymbol{\mu}_{d}} \log p\left(\boldsymbol{\mu}_{r} \mid \boldsymbol{\mu}_{d}\right)\right|_{\boldsymbol{\mu}_{d}^{\star}}
\end{array}\right)\right] .
$$

Obviously, if we assume $p\left(\boldsymbol{\mu}_{r} \mid \boldsymbol{\mu}_{d}\right)=p\left(\boldsymbol{\mu}_{r}\right)$ in this expression, we straightforwardly reobtain Eqn. (3).

Based on this structure, one now has to prove that there is still an inequality, i.e., a lower bound on the MSE,

$$
\mathbb{E}_{\mathbf{y}, \boldsymbol{\mu}_{r} \mid \boldsymbol{\mu}_{d}^{\star}}\left[\left.(\hat{\boldsymbol{\mu}}(\mathbf{y})-\boldsymbol{\mu})(\hat{\boldsymbol{\mu}}(\mathbf{y})-\boldsymbol{\mu})^{T}\right|_{\boldsymbol{\mu}_{d}^{\star}}\right] \geq \mathbf{H}^{-1}\left(\boldsymbol{\mu}_{d}^{\star}\right),
$$

when $\mathbf{H}\left(\boldsymbol{\mu}_{d}^{\star}\right)$ is given by Eqn. (8).

Proof: Following the idea of [4] to prove the inequality (1), one defines a vector $\mathbf{h}$ such that

$$
\mathbf{h}=\left(\begin{array}{c}
\left.\nabla_{\boldsymbol{\mu}} \log p\left(\mathbf{y}, \boldsymbol{\mu}_{r} \mid \boldsymbol{\mu}_{d}\right)\right|_{\boldsymbol{\mu}_{d}^{\star}} \\
\hat{\boldsymbol{\mu}}(\mathbf{y})-\left.\boldsymbol{\mu}\right|_{\boldsymbol{\mu}_{d}^{\star}}
\end{array}\right),
$$

where $\nabla_{\boldsymbol{\mu}}=\left(\begin{array}{ccc}\frac{\partial}{\partial[\boldsymbol{\mu}]_{1}} & \cdots & \frac{\partial}{\partial[\boldsymbol{\mu}]_{n}}\end{array}\right)^{T}$.

Consequently, the non-negative definite matrix $\mathbf{G}\left(\boldsymbol{\mu}_{d}^{\star}\right)=\mathbb{E}_{\mathbf{y}, \boldsymbol{\mu}_{r} \mid \boldsymbol{\mu}_{d}^{\star}}\left[\mathbf{h} \mathbf{h}^{T}\right]$ can be decomposed as the following block matrix

$$
\mathbf{G}\left(\boldsymbol{\mu}_{d}^{\star}\right)=\left(\begin{array}{cc}
\mathbf{H}\left(\boldsymbol{\mu}_{d}^{\star}\right) & \mathbf{L}\left(\boldsymbol{\mu}_{d}^{\star}\right) \\
\mathbf{L}^{T}\left(\boldsymbol{\mu}_{d}^{\star}\right) & \mathbf{R}\left(\boldsymbol{\mu}_{d}^{\star}\right)
\end{array}\right),
$$

where $\mathbf{R}\left(\boldsymbol{\mu}_{d}^{\star}\right)$ is the covariance matrix of $\hat{\boldsymbol{\mu}}(\mathbf{y})$, i.e.,

$$
\mathbf{R}\left(\boldsymbol{\mu}_{d}^{\star}\right)=\mathbb{E}_{\mathbf{y}, \boldsymbol{\mu}_{r} \mid \boldsymbol{\mu}_{d}^{\star}}\left[\left.(\hat{\boldsymbol{\mu}}(\mathbf{y})-\boldsymbol{\mu})(\hat{\boldsymbol{\mu}}(\mathbf{y})-\boldsymbol{\mu})^{T}\right|_{\boldsymbol{\mu}_{d}^{\star}}\right]
$$

and, where $\mathbf{L}\left(\boldsymbol{\mu}_{d}^{\star}\right)$ is given by

$$
\mathbf{L}\left(\boldsymbol{\mu}_{d}^{\star}\right)=\mathbb{E}_{\mathbf{y}, \boldsymbol{\mu}_{r} \mid \boldsymbol{\mu}_{d}^{\star}}\left[\left.\nabla_{\boldsymbol{\mu}} \log p\left(\mathbf{y}, \boldsymbol{\mu}_{r} \mid \boldsymbol{\mu}_{d}\right)\right|_{\boldsymbol{\mu}_{d}^{\star}}\left(\hat{\boldsymbol{\mu}}(\mathbf{y})-\left.\boldsymbol{\mu}\right|_{\boldsymbol{\mu}_{d}^{\star}}\right)^{T}\right] .
$$

Since $\mathbf{G}\left(\boldsymbol{\mu}_{d}^{\star}\right) \geq 0$, its Schur complement satisfies

$$
\mathbf{R}\left(\boldsymbol{\mu}_{d}^{\star}\right) \geq \mathbf{L}^{T}\left(\boldsymbol{\mu}_{d}^{\star}\right) \mathbf{H}^{-1}\left(\boldsymbol{\mu}_{d}^{\star}\right) \mathbf{L}\left(\boldsymbol{\mu}_{d}^{\star}\right) .
$$

It is straightforward to show that, for an unbiased estimator w.r.t. the pdf $p\left(\mathbf{y}, \boldsymbol{\mu}_{r} \mid \boldsymbol{\mu}_{d}^{\star}\right), \mathbf{L}\left(\boldsymbol{\mu}_{d}^{\star}\right)=\mathbf{I}_{n \times n}$. Consequently, the inequality (9) is proved and $\mathbf{H}^{-1}\left(\boldsymbol{\mu}_{d}^{\star}\right)$ is a lower bound on the MSE. 


\section{HCRB FOR A DYNAMICAL PHASE ESTIMATION PROBLEM}

In [6], we have proposed a closed-form expression of the Bayesian CRB for the estimation of the phase offset for a BPSK transmission in a non data-aided context. In this section, we extend these previous results by providing a closed-form expression of the HCRB for the estimation of the phase offset and also of the linear drift. In this more realistic scenario, we show that we have to take into account the statistical dependence between the parameters and, consequently, the HCRB given by Eqn. (3) is not adapted to this problem.

\section{A. Observation and state models}

We consider a linearly modulated signal, obtained by applying to a square-root Nyquist transmit filter an unknown symbol sequence $\mathbf{a}=\left(a_{1} \cdots a_{K}\right)^{T}$ taken from a unit energy BPSK constellation. The signal is transmitted over an additive white Gaussian noise channel. The output signal is sampled at the symbol rate which yields to the observations

$$
y_{k}=a_{k} e^{j \theta_{k}}+n_{k} \quad \text { with } k=1 \ldots K
$$

where $\left\{n_{k}\right\}$ is a sequence of i.i.d., circular, zero mean complex Gaussian noise variables with variance $\sigma_{n}^{2}$. We consider that the system operates in a non Data-Aided synchronization mode, i.e., the transmitted symbols are i.i.d. with $P_{r}\left(a_{k}= \pm 1\right)=\frac{1}{2}$.

In practice, several sources of distortions affect the phase. An efficient model representing these effects is the so-called Brownian phase with a linear drift widely studied in the literature (see, e.g., [7] [8] [9]). This model takes into account a constant frequency shift between the oscillators of the transmitter and of the receiver, the uncertainities due to clocks, and, the jitters of oscillators. The Brownian phase model with a linear drift is given as follows

$$
\theta_{k}=\theta_{k-1}+\xi+w_{k} \quad \text { with } k=2 \ldots K
$$

where, for any index $k,\left\{\theta_{k}\right\}$ is the sequence of phases to be estimated, $\xi$ represents the deterministic unknown linear drift with true value $\xi^{\star}$, and where $\left\{w_{k}\right\}$ is an i.i.d. sequence of centered Gaussian random variables with known variance $\sigma_{w}^{2}$.

The parameter vector of interest is then made up of both random and deterministic parameters $\boldsymbol{\mu}=\left(\boldsymbol{\mu}_{r}^{T} \mu_{d}\right)^{T}$ where $\boldsymbol{\mu}_{r}=\boldsymbol{\theta}=\left(\theta_{1} \cdots \theta_{K}\right)^{T}$ and $\mu_{d}=\xi$. Moreover, from Eqn. (16), it is clear that $p\left(\boldsymbol{\theta} \mid \xi^{\star}\right) \neq p(\boldsymbol{\theta})$.

\section{B. Derivation of the $H C R B$}

For notational convenience, we drop the dependence of the different matrices on $\mu_{d}^{\star}=\xi^{\star}$ in the remainder of this paper. From Eqn. (8), the HIM $\mathbf{H}$ can be rewritten into a block matrix $\mathbf{H}=\left(\begin{array}{ll}\mathbf{H}_{11} & \mathbf{h}_{12} \\ \mathbf{h}_{21} & H_{22}\end{array}\right)$, where,

$$
\left\{\begin{array}{l}
\mathbf{H}_{11}=\mathbb{E}_{\mathbf{y}, \boldsymbol{\theta} \mid \xi^{\star}}\left[-\left.\Delta_{\boldsymbol{\theta}}^{\boldsymbol{\theta}} \log p(\mathbf{y} \mid \boldsymbol{\theta}, \xi)\right|_{\xi^{\star}}\right]+\mathbb{E}_{\boldsymbol{\theta} \mid \xi^{\star}}\left[-\Delta_{\boldsymbol{\theta}}^{\boldsymbol{\theta}} \log p\left(\boldsymbol{\theta} \mid \xi^{\star}\right)\right] \\
\mathbf{h}_{12}=\mathbf{h}_{21}^{T}=\mathbb{E}_{\mathbf{y}, \boldsymbol{\theta} \mid \xi^{\star}}\left[-\left.\Delta_{\boldsymbol{\theta}}^{\xi} \log p(\mathbf{y} \mid \boldsymbol{\theta}, \xi)\right|_{\xi^{\star}}\right]+\mathbb{E}_{\boldsymbol{\theta} \mid \xi^{\star}}\left[-\left.\Delta_{\boldsymbol{\theta}}^{\xi} \log p(\boldsymbol{\theta} \mid \xi)\right|_{\xi^{\star}}\right] \\
H_{22}=\mathbb{E}_{\mathbf{y}, \boldsymbol{\theta} \mid \xi^{\star}}\left[-\left.\Delta_{\xi}^{\xi} \log p(\mathbf{y} \mid \boldsymbol{\theta}, \xi)\right|_{\xi^{\star}}\right]+\mathbb{E}_{\boldsymbol{\theta} \mid \xi^{\star}}\left[-\left.\Delta_{\xi}^{\xi} \log p(\boldsymbol{\theta} \mid \xi)\right|_{\xi^{\star}}\right]
\end{array}\right.
$$


These blocks only depend on the $\log$-likelihoods $\log p\left(\mathbf{y} \mid \boldsymbol{\theta}, \xi^{\star}\right)$ and $\log p\left(\boldsymbol{\theta} \mid \xi^{\star}\right)$. Let us set $\mathbf{y}=\left(y_{1} \cdots y_{K}\right)^{T}$ and assume that the initial phase $\theta_{1}$ does not depend on $\xi$, i.e., $p\left(\theta_{1} \mid \xi^{\star}\right)=p\left(\theta_{1}\right)$. Using Eqn. (15) and (16), i.e., the Gaussian nature of the noise and the equiprobability of the symbols, one has

$$
\left\{\begin{array}{l}
\log p\left(\mathbf{y} \mid \boldsymbol{\theta}, \xi^{\star}\right)=\sum_{k=1}^{K}\left(-\log \left(\pi \sigma_{n}^{2}\right)-\frac{1+\left\|y_{k}\right\|^{2}}{\sigma_{n}^{2}}+\log \left(\cosh \left(\frac{2}{\sigma_{n}^{2}} \Re\left\{y_{k} e^{-j \theta_{k}}\right\}\right)\right)\right) \\
\log p\left(\boldsymbol{\theta} \mid \xi^{\star}\right)=\log p\left(\theta_{1}\right)+(K-1) \log \left(\frac{1}{\sqrt{2 \pi} \sigma_{w}}\right)-\sum_{k=2}^{K} \frac{\left(\theta_{k}-\theta_{k-1}-\xi^{\star}\right)^{2}}{2 \sigma_{w}^{2}}
\end{array}\right.
$$

- Expression of $\mathbf{H}_{11}$ : assuming that we have no prior knowledge, i.e., $\mathbb{E}_{\theta_{1}}\left[\Delta_{\theta_{1}}^{\theta_{1}} \log p\left(\theta_{1}\right)\right]=0$, it is shown in [6] (due to the order one Markov structure exhibited by Eqn. (16)) that $\mathbf{H}_{11}$ takes the following tridiagonal structure

$$
\mathbf{H}_{11}=b\left(\begin{array}{ccccc}
A+1 & 1 & 0 & \cdots & 0 \\
1 & A & 1 & \ddots & \vdots \\
0 & \ddots & \ddots & \ddots & 0 \\
\vdots & \ddots & 1 & A & 1 \\
0 & \ldots & 0 & 1 & A+1
\end{array}\right)
$$

where $b=-1 / \sigma_{w}^{2}$, and, where $A=-\sigma_{w}^{2} J_{D}-2$ with $J_{D}=\mathbb{E}_{\mathbf{y}, \boldsymbol{\theta} \mid \xi^{\star}}\left[-\Delta_{\theta_{k}}^{\theta_{k}} \log p\left(y_{k} \mid \theta_{k}, \xi^{\star}\right)\right]$.

- Expression of $\mathbf{h}_{12}$ : since, from Eqn. (18), $\log p\left(\mathbf{y} \mid \boldsymbol{\theta}, \xi^{\star}\right)$ is independent of $\xi^{\star},\left.\Delta_{\theta}^{\xi} \log p(\mathbf{y} \mid \boldsymbol{\theta}, \xi)\right|_{\xi^{\star}}=0$. Consequently,

$$
\mathbf{h}_{12}=\mathbb{E}_{\boldsymbol{\theta} \mid \xi^{\star}}\left[-\left.\Delta_{\boldsymbol{\theta}}^{\xi} \log p(\boldsymbol{\theta} \mid \xi)\right|_{\xi^{\star}}\right]
$$

Using the state model, we have

$$
\left\{\begin{array}{l}
\left.\Delta_{\xi}^{\theta_{1}} \log p(\boldsymbol{\theta} \mid \xi)\right|_{\xi^{\star}}=-\frac{1}{\sigma_{w}^{2}}, \\
\left.\Delta_{\xi}^{\theta_{K}} \log p(\boldsymbol{\theta} \mid \xi)\right|_{\xi^{\star}}=\frac{1}{\sigma_{w}^{2}}, \\
\left.\Delta_{\xi}^{\theta_{k}} \log p(\boldsymbol{\theta} \mid \xi)\right|_{\xi^{\star}}=0 \text { for } k \in\{2, \ldots, K-1\} .
\end{array}\right.
$$

Applying the expectation operator $\mathbb{E}_{\boldsymbol{\theta} \mid \xi^{\star}}[$.$] , we obtain$

$$
\mathbf{h}_{12}=\left(\begin{array}{ccc}
\frac{1}{\sigma_{w}^{2}} & \mathbf{0}_{1 \times K-2} & -\frac{1}{\sigma_{w}^{2}}
\end{array}\right)^{T}
$$

- Expression of $H_{22}$ : since, from Eqn. (18), $\log p\left(\mathbf{y} \mid \boldsymbol{\theta}, \xi^{\star}\right)$ is independent of $\xi^{\star},\left.\Delta_{\xi}^{\xi} \log p(\mathbf{y} \mid \boldsymbol{\theta}, \xi)\right|_{\xi^{\star}}=0$. Consequently,

$$
H_{22}=\mathbb{E}_{\boldsymbol{\theta} \mid \xi^{\star}}\left[-\left.\Delta_{\xi}^{\xi} \log p(\boldsymbol{\theta} \mid \xi)\right|_{\xi^{\star}}\right]=\frac{K-1}{\sigma_{w}^{2}} .
$$

- Expression of the HCRB: we now give the expression of $\mathbf{H}^{-1}$ which bounds the MSE. Thanks to the blockmatrix inversion formula, we have

$$
\mathbf{H}^{-1}=\left(\begin{array}{cc}
\mathbf{H}_{11}^{-1}+\mathbf{V}_{K} & -\frac{1}{\lambda} \mathbf{H}_{11}^{-1} \mathbf{h}_{12} \\
-\frac{1}{\lambda} \mathbf{h}_{12}^{T} \mathbf{H}_{11}^{-1} & \frac{1}{\lambda}
\end{array}\right)
$$

where $\lambda=\frac{K-1}{\sigma_{w}^{2}}-\mathbf{h}_{12}^{T} \mathbf{H}_{11}^{-1} \mathbf{h}_{12}$ and $\mathbf{V}_{K}=\frac{1}{\lambda} \mathbf{H}_{11}^{-1} \mathbf{h}_{12} \mathbf{h}_{12}^{T} \mathbf{H}_{11}^{-1}$. 
We start to compute $\lambda$ corresponding to the inverse of the minimal bound on the MSE of $\xi$. Due to the particular structure of matrices $\mathbf{H}_{11}$ and $\mathbf{h}_{12}$ (Eqn. (19) and (22)), we obtain

$$
\lambda=\frac{K-1}{\sigma_{w}^{2}}-\frac{2}{\sigma_{w}^{4}}\left(\left[\mathbf{H}_{11}^{-1}\right]_{1,1}-\left[\mathbf{H}_{11}^{-1}\right]_{1, K}\right) .
$$

From Eqn. (19), thanks to the cofactor expression in the matrix inversion formula we have for any index $k$, $\left[\mathbf{H}_{11}^{-1}\right]_{1, k}=\frac{b^{k-1}}{\left|\mathbf{H}_{11}\right|}\left(d_{K-k}+b d_{K-k-1}\right)$ where $d_{k}$ is the determinant of the following $k \times k$ matrix $\mathbf{D}_{k}$

$$
\mathbf{D}_{k}=b\left(\begin{array}{ccccc}
A & 1 & 0 & \cdots & 0 \\
1 & A & 1 & \ddots & \vdots \\
0 & \ddots & \ddots & \ddots & 0 \\
\vdots & \ddots & 1 & A & 1 \\
0 & \cdots & 0 & 1 & A
\end{array}\right) .
$$

The sequence $\left\{d_{k}\right\}$ satisfies the following recursive equation $d_{k}=A b d_{k-1}-b^{2} d_{k-2}$ with $d_{0}=1$ and $d_{1}=b A$. $d_{k}$ can thus be written as $d_{k}=\rho_{1}\left(r_{1}\right)^{k}+\rho_{2}\left(r_{2}\right)^{k}$ where $r_{1}, r_{2}, \rho_{1}$ and $\rho_{2}$ are given by

$$
\left\{\begin{array}{cc}
r_{1}=\frac{b}{2}\left(A+\sqrt{A^{2}-4}\right), & r_{2}=\frac{b}{2}\left(A-\sqrt{A^{2}-4}\right), \\
\rho_{1}=\frac{\sqrt{A^{2}-4}+A}{2 \sqrt{A^{2}-4}}, & \rho_{2}=\frac{\sqrt{A^{2}-4}-A}{2 \sqrt{A^{2}-4}} .
\end{array}\right.
$$

Consequently,

$$
\left[\mathbf{H}_{11}^{-1}\right]_{1, k}=\frac{b^{k-1}}{\left|\mathbf{H}_{11}\right|}\left(\rho_{1} r_{1}^{K-k-1}\left(r_{1}+b\right)+\rho_{2} r_{2}^{K-k-1}\left(r_{2}+b\right)\right)
$$

and

$$
\lambda=\frac{K-1}{\sigma_{w}^{2}}-\frac{2}{\sigma_{w}^{4}\left|\mathbf{H}_{11}\right|}\left(\rho_{1} r_{1}^{K-2}\left(r_{1}+b\right)+\rho_{2} r_{2}^{K-2}\left(r_{2}+b\right)-b^{K-1}\right) .
$$

From the definition of $\mathbf{V}_{K}$, we have

$$
\left[\mathbf{V}_{K}\right]_{k, k}=\frac{1}{\lambda \sigma_{w}^{4}}\left(\left[\mathbf{H}_{11}^{-1}\right]_{1, k}-\left[\mathbf{H}_{11}^{-1}\right]_{1, K+1-k}\right)^{2}
$$

Using Eqn. (24), (28), and (30), we obtain, for any index $k$, the analytical expression of the HCRB diagonal elements

$$
\begin{gathered}
{\left[\mathbf{H}^{-1}\right]_{k, k}=\frac{1}{\left|\mathbf{H}_{11}\right|}\left[\rho_{1}^{2}\left(b+r_{1}\right)^{2} r_{1}^{K-3}+\rho_{2}^{2}\left(b+r_{2}\right)^{2} r_{2}^{K-3}-\frac{b^{2}}{A-2}\left(r_{1}^{k-2} r_{2}^{K-k-1}+r_{1}^{K-k-1} r_{2}^{k-2}\right)\right]} \\
+\frac{1}{\lambda \sigma_{w}^{4}\left|\mathbf{H}_{11}\right|^{2}}\left[b^{k-1}\left(\rho_{1}\left(r_{1}\right)^{K-k-1}\left(b+r_{1}\right)+\rho_{2}\left(r_{2}\right)^{K-k-1}\left(b+r_{2}\right)\right)\right. \\
\left.+b^{K-k}\left(\rho_{1}\left(r_{1}\right)^{k-2}\left(b+r_{1}\right)+\rho_{2}\left(r_{2}\right)^{k-2}\left(b+r_{2}\right)\right)\right]^{2} .
\end{gathered}
$$

Remark: Note that, if Eqn. (3) was used instead of Eqn. (8), the HIM would not be invertible.

\section{Simulation results}

We now illustrate the behavior of the HCRB versus the Signal-to-Noise Ratio (SNR) defined by $\frac{1}{\sigma_{n}^{2}}$. We consider a block of $K=40$ BPSK transmitted symbols. For two distinct phase-noise variances $\left(\sigma_{w}^{2}=0.1 \mathrm{rad}^{2}\right.$ and $\sigma_{w}^{2} \rightarrow$ $0 \operatorname{rad}^{2}$ ), Figure 1 superimposes on one side the HCRB (see Eqn. (31)), the Data-Aided $\operatorname{HCRB}\left(J_{D}=\frac{2}{\sigma_{n}^{2}}\right)$, and 
the BCRB (see Eqn. (21) in [6]) on $\theta_{K}$. For the same scenario, Figure 2 superimposes on one side the HCRB (see Eqn. (25)) and the Data-Aided HCRB on $\xi$.

- At high SNR, we first notice that $\mathrm{HCRB}_{\xi}$ converges to its horizontal asymptote given by $\frac{\sigma_{w}^{2}}{K-1}$ which is the standard CRB when $\boldsymbol{\theta}$ is assumed to be known. The observation noise compared to the phase noise is then not significant enough to disturb the estimation of $\xi$; consequently $\mathrm{HCRB}_{\xi}$ depends only on the phase noise and on the number of observations. Concerning the bounds on $\theta_{K}, \mathrm{HCRB}_{\theta_{k}}$ and $\mathrm{BCRB}_{\theta_{k}}$ both have the same asymptote given by $\frac{\sigma_{n}^{2}}{2}$ which is the Modified CRB (MCRB) for one observation (see [10]). It means that, at high SNR, the observation $y_{K}$ is self-sufficient to estimate $\theta_{K}$ and the error on $\xi$ does not disturb the performance on $\theta_{K}$. Moreover, the HCRB logically tends to the Data-Aided HCRB.

- For median $\mathrm{SNR}, \mathrm{HCRB}_{\theta_{K}}$ and $\mathrm{HCRB}_{\xi}$ leave their respective asymptote. $\mathrm{HCRB}_{\theta_{K}}$ is still lower bounded by the BCRB and upper bounded by the high-SNR asymptote. This stems from the fact that taking into account a block of observations instead of one observation necessarily improves the performance. However, for large $\sigma_{w}^{2}$ values (e.g., $\left.\sigma_{w}^{2}=0.1 \mathrm{rad}^{2}\right), \mathrm{HCRB}_{\theta_{K}}$ stays close to the MCRB because the correlation between the phase offsets $\theta_{k}$ is less significant than the information brought by the observation $y_{K}$. Moreover, when $\sigma_{w}^{2}$ tends to $0, \mathrm{HCRB}_{\theta_{K}}$ is above the BCRB because performance is now limited by the accuracy on the parameter $\xi$.

- At low SNR, $n_{k}$ is preponderant compared to $w_{k}$. Both $\mathrm{HCRB}_{\xi}$ and $\mathrm{HCRB}_{\theta_{K}}$ do not depend on $\sigma_{w}^{2}$ : the lack of knowledge on $\xi$ directly affects the estimation on $\theta_{K}$. As expected, the knowledge of the symbols (Data-Aided HCRB) leads to a better estimation of $\boldsymbol{\theta}$ and $\xi$.

\section{CONCLUSION}

In this paper, we have studied the hybrid Cramér-Rao bound when the random and the deterministic parts of the parameter vector are statistically dependent. We have applied this bound in order to evaluate the performance of a dynamical phase estimator where the linear drift is unknown in a non data-aided context. In particular, we have illustrated the effect of this unknown linear drift on the phase estimation performance.

\section{REFERENCES}

[1] H. Cramér, Mathematical Methods of Statistics, ser. Princeton Mathematics. New-York: Princeton University Press, Sept. 1946 , vol. 9.

[2] H. L. Van Trees, Detection, Estimation and Modulation Theory. New-York, NY, USA: John Wiley \& Sons, 1968, vol. 1.

[3] Y. Rockah and P. Schultheiss, "Array shape calibration using sources in unknown locations-part I: Far-field sources," IEEE Transactions on Acoustics, Speech, and Signal Processing, vol. 35, no. 3, pp. 286-299, Mar. 1987.

[4] H. Messer, "The hybrid Cramér-Rao lower bound - from practice to theory," in Proc. of IEEE Workshop on Sensor Array and Multi-channel Processing (SAM), Waltham, MA, USA, July 2006, pp. 304-307.

[5] A. M. Guarnieri and S. Tebaldini, "Hybrid Cramér-Rao bounds for crustal displacement field estimators in SAR interferometry," IEEE Signal Processing Letters, vol. 14, no. 12, pp. 1012-1015, Dec. 2007.

[6] S. Bay, C. Herzet, J.-M. Brossier, J.-P. Barbot, and B. Geller, "Analytic and asymptotic analysis of Bayesian Cramér-Rao bound for dynamical phase offset estimation,” IEEE Transactions on Signal Processing, vol. 56, no. 1, pp. 61-70, Jan. 2008.

[7] P.-O. Amblard, J.-M. Brossier, and E. Moisan, "Phase tracking: what do we gain from optimality? Particle filtering versus phase-locked loops," Signal Processing, vol. 83, no. 1, pp. 151-167, Jan. 2003.

[8] J. A. McNeill, “Jitter in ring oscillators," Ph.D. dissertation, Boston University, 1994. 


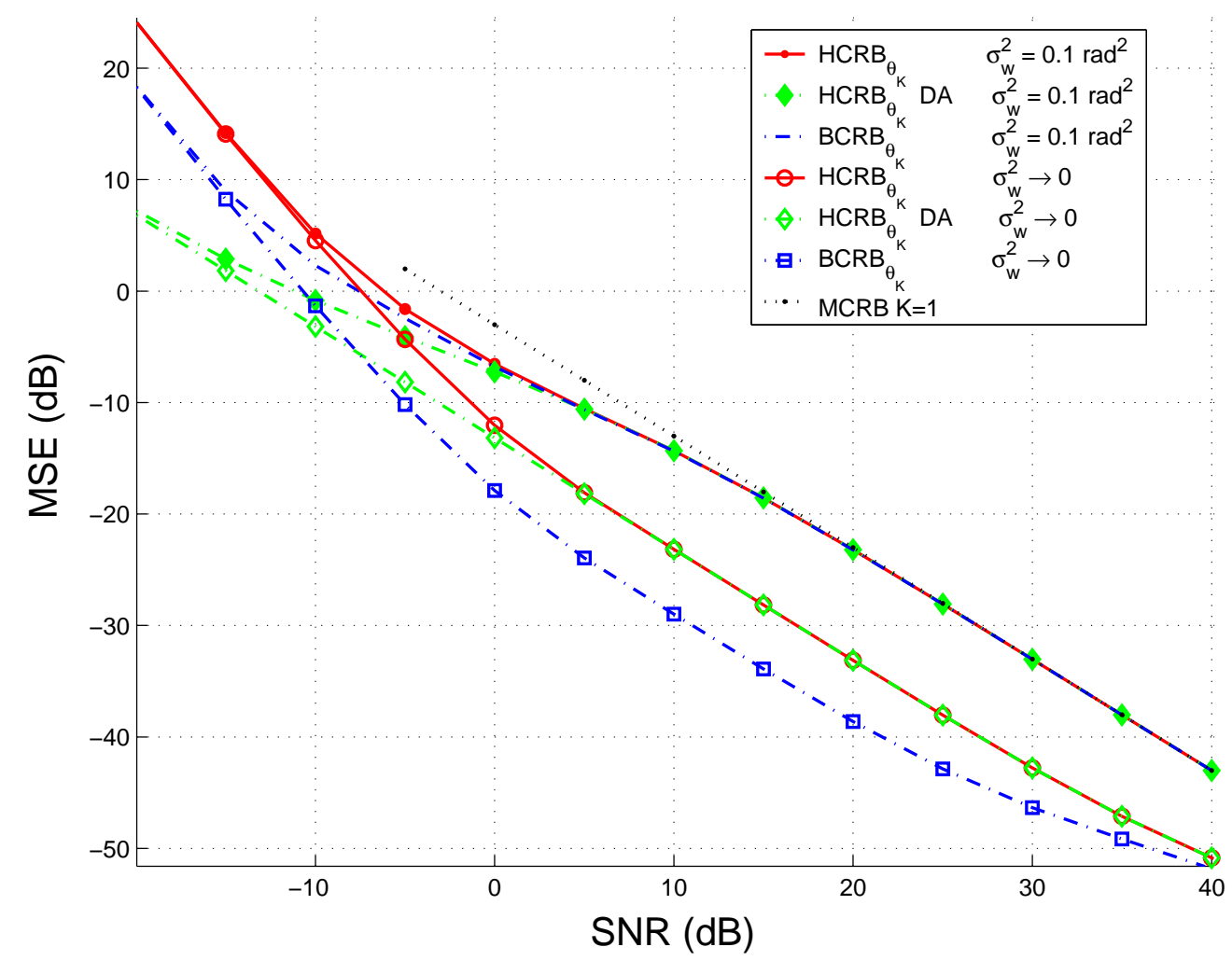

Fig. 1. Bounds on $\theta_{K}$ versus the SNR ( $K=40$ observations, $\sigma_{w}^{2}=0.1 \mathrm{rad}^{2}$, and $\sigma_{w}^{2} \rightarrow 0 \mathrm{rad}^{2}, J_{D}$ evaluated over $10^{8}$ Monte-Carlo trials).

[9] A. Demir, A. Mehrotra, and J. Roychowdhury, "Phase noise in oscillators: a unifying theory and numerical methods for characterization," IEEE Trans. on Circuits and Systems, vol. 47, pp. 655-674, May 2000.

[10] M. Moeneclaey, "On the true and the modified Cramér-Rao bounds for the estimation of a scalar parameter in the presence of nuisance parameters," IEEE Transactions on Communications, vol. 46, no. 11, pp. 1536-1544, Nov. 1998. 


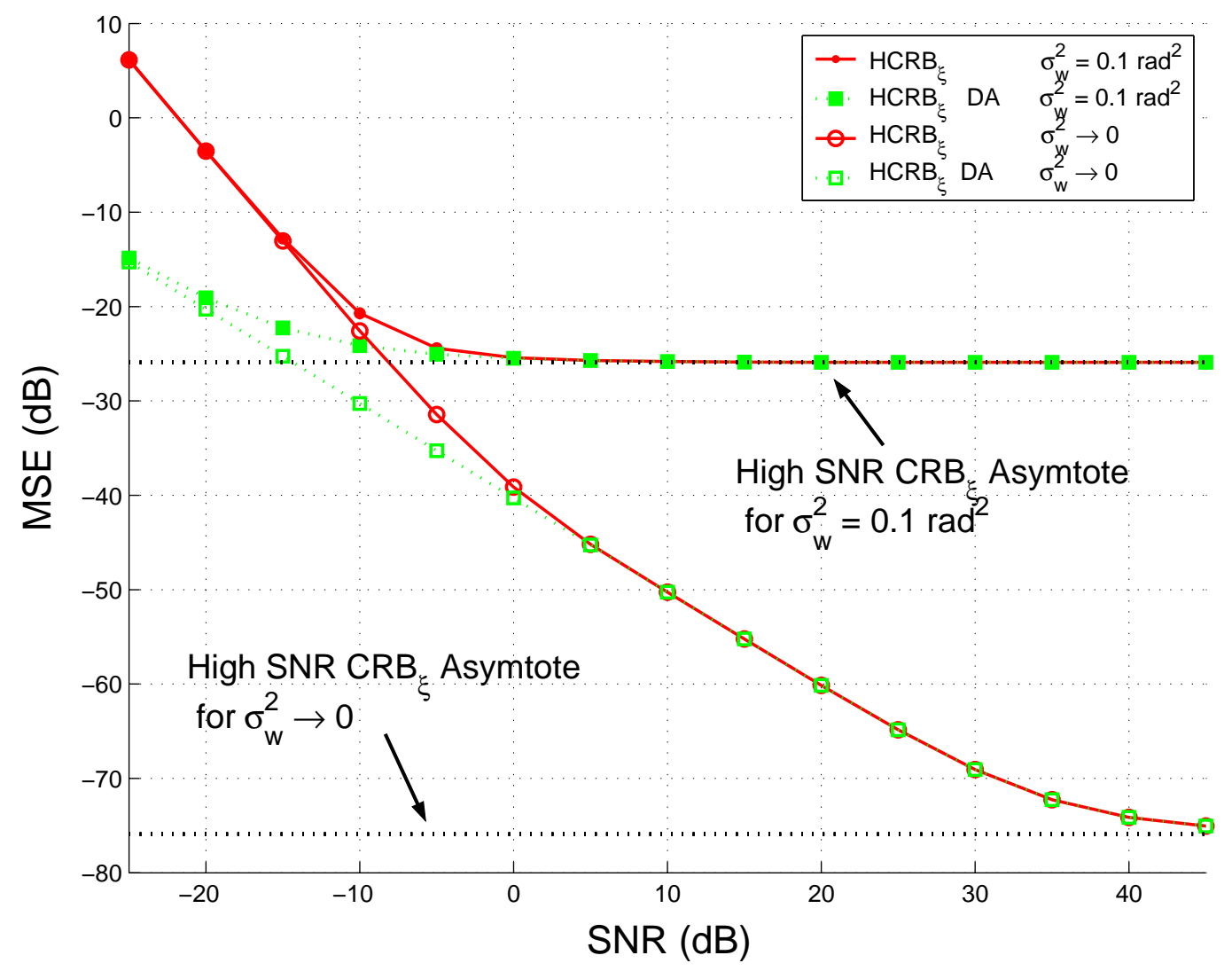

Fig. 2. Bounds on $\xi$ versus the SNR ( $K=40$ observations, $\sigma_{w}^{2}=0.1 \mathrm{rad}^{2}$ and $\sigma_{w}^{2} \rightarrow 0 \mathrm{rad}^{2}, J_{D}$ evaluated over $10^{8}$ Monte-Carlo trials). 